\title{
Modello numerico per la simulazione e l'ottimizzazione di controlli non distruttivi con ultrasuoni
}

\author{
A. Pantano, D. Cerniglia \\ Università degli Studi di Palermo, Dipartimento di Meccanica, Viale delle Scienæe, 90128, Palermo \\ apantano@dima.unipa.it,cerniglia@dima.unipa.it
}

RIASsUNTO. I controlli non distruttivi basati sull'impiego di ultrasuoni sono ampiamente usati per la loro efficacia e affidabilità nel rilevamento di difetti. La generazione di onde ultrasonore e la propagazione in strutture di forma non regolare sono difficili da analizzare, soprattutto se la sorgente impiegata è un laser. Le tecniche numeriche per la simulazione del fenomeno reperibili in letteratura mostrano limiti di applicabilità per frequenze nel campo dei $\mathrm{MHz}$ e lunghezze d'onda molto corte. In questo lavoro presentiamo un metodo numerico in grado di risolvere accuratamente ed efficientemente problemi di generazione di onde ultrasonore tramite laser, con frequenze nel range dei $\mathrm{MHz}$, e di propagazione in corpi relativamente estesi. La ricezione viene simulata con la propagazione degli ultrasuoni in aria, al fine di poter ottimizzare la configurazione completa per controlli non distruttivi con ultrasuoni senza contatto. Diverse configurazioni di ispezione sono state prima simulate tramite l'analisi numerica e poi riprodotte sperimentalmente per confrontare i risultati.

ABSTRACT. Non-destructive testings (NDT) based on ultrasonic methods are extensively used for their effectiveness and reliability in detecting defects. The generation of ultrasonic waves and propagation in nonregular geometries are difficult to analyze, especially if the source used is a laser.

Numerical techniques to simulate the phenomenon found in literature have proved to be limited in their applicability by the frequencies in the $\mathrm{MHz}$ range and very short wavelengths. In this paper we present a numerical method to accurately and efficiently solve problems of laser-generated ultrasounds, with frequencies in the $\mathrm{MHz}$ range, and propagation in relatively large bodies. Detection is simulated with the propagation of ultrasounds in air, to optimize the complete configuration for non-contact ultrasonic NDT. Different configurations of inspections have been first simulated using numerical analysis and then reproduced experimentally to compare the results.

Parole Chiave. Controlli non distruttivi, Metodi numerici.

\section{INTRODUZIONE}

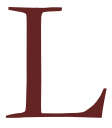

e tecniche di ispezione ad ultrasuoni, ampiamente usate per il rilievo di difetti, sono in continua evoluzione soprattutto per l'introduzione e lo sviluppo di nuove sonde e metodi. Le tecniche convenzionali si basano generalmente sull'uso di trasduttori a contatto, in cui l'ispezione dipende dalle condizioni di contatto (pressione, mezzo di accoppiamento, area di contatto). Gli sviluppi degli ultimi decenni sono diretti alla considerazione di sonde e metodi senza contatto con la struttura [1-2]. L'uso del laser, sia come sorgente di ultrasuoni che come dispositivo di ricezione, è in continuo sviluppo poiché consente l'ispezione remota, realizzabile in dinamico e automatizzabile guidando il raggio laser attraverso bracci ottici [3-5].

La propagazione di onde ultrasonore generate tramite laser in strutture di forma non regolare è un fenomeno difficile da analizzare, considerato che le onde includono i modi longitudinali, trasversali e di superficie e che propagando attraverso 
la struttura ed interagendo con difetti, spigoli e bordi in geometrie complesse provocano riflessione e conversione di modo. In aggiunta, le interferenze tra le onde contribuiscono a generare una distribuzione di onde ultrasonore molto complessa.

La propagazione di onde ultrasonore può essere studiata analiticamente risolvendo la equazioni del moto con opportune condizioni al contorno. Uno dei primi lavori sulla generazione di onde termoeleastiche in un corpo indotte da una sorgente di calore superficiale è stata prodotta da White [6] nei primi anni sessanta. Il suo sforzo può essere considerato un modello di prima generazione non in grado di predire le onde di taglio e superficiali generate dall'impulso laser. Il modello di seconda generazione, definito a sorgente puntuale, fu ideato inizialmente da Scruby et al. [7] il quale identificò che in regime termoelastico la regione riscaldata dal laser agisce come centro di espansione superficiale. Più tardi Rose [8] fornì al metodo una base matematica più rigorosa. Tuttavia la teoria a sorgente puntuale aveva dei limiti, in particolare non considerava diffusione termica. McDonald [9] sviluppò un modello di terza generazione, che tiene in considerazione la diffusione termica e la forma finita dell'impulso laser, riportando ottima corrispondenza tra i risultati sperimentali e teorici. I modelli analitici, tuttavia, diventano intrattabili per geometrie complicate o per provini con difetti. Un altro approccio per ottenere una soluzione per problemi relativi a onde ultrasonore generate tramite laser è il metodo numerico agli elementi finiti (FE), ampiamente scelto dalla comunità scientifica come strumento in molti studi sulla propagazione di onde ultrasonore [10-22].

La modellazione tramite elementi finiti consente di studiare l'interazione di onde con difetti ma richiede il rispetto di regole molto stringenti sulla discretizzazione spaziale e temporale. Ne consegue che se le dimensioni del pezzo sono significative e le frequenze in gioco sono alte il numero dei gradi di libertà del modello tende a diventare molto elevato, rendendo spesso impossibile risolvere problemi reali di propagazione di onde. Gli ultrasuoni generati tramite laser hanno frequenze nell'ordine dei $\mathrm{MHz}$ richiedendo incrementi di tempo molto piccoli tra le soluzioni. Inoltre queste alte frequenze si abbinano a delle lunghezze d'onda molto corte, e siccome per una risoluzione spaziale ragionevole delle onde la dimensione degli elementi finiti deve essere al massimo $1 / 10$ della più piccola lunghezza d'onda da analizzare [11] gli elementi devono essere molto piccoli. Questo spiega perchè nella vasta letterature sull'argomento non si sono trovati lavori riguardanti la propagazione di ultrasuoni nel range di frequenza dei $\mathrm{MHz}$ viaggianti in corpi più larghi di pochi centimetri nelle tre direzioni. La comunità scientifica potrebbe essere portata a concludere che questa classe di problemi non può essere studiata tramite l'uso del metodo degli elementi finiti eseguito su moderne workstations.

In questo lavoro viene presentato un metodo numerico che permette di simulare la generazione degli ultrasuoni tramite laser, con frequenze nel range dei $\mathrm{MHz}$, e la loro propagazione in corpi relativamente estesi. Al fine di simulare una configurazione di ispezione senza contatto, viene introdotta la simulazione della propagazione in aria.

\section{MODELlO NUMERICO}

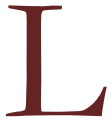

a letteratura sulla modellazione agli elementi finiti della propagazione di onde ultrasonore mostra un esteso uso dell'integrazione implicita delle equazioni del moto. Tuttavia, mentre vi sono diverse classi di problemi dove l'integrazione implicita è computazionalmente più efficiente dell'integrazione esplicita, ci sono ragioni tecniche che rendono l'integrazione esplicita superiore nel simulare problemi di propagazione di onde ultrasonore. I problemi di propagazione di onde sono caratterizzati da frequenze molto elevate. I periodi temporali di interesse sono molto brevi; spesso si osserva il fenomeno per il tempo necessario al fronte d'onda ad attraversare l'intera struttura. Durante il transitorio in genere si analizza la propagazione delle onde di tensione.

In un'analisi dinamica di tipo esplicito la dimensione dell'incremento temporale è piccola, perché l'operatore alla differenze centrate è condizionalmente stabile e richiede che all'interno di un incremento temporale l'informazione non si propaghi attraverso più di un elemento [23].

Nel caso della propagazione di onde questo significa che l'incremento temporale deve essere più breve del tempo richiesto ad un'onda di tensione per attraversare l'elemento più piccolo nel modello, quindi se gli elementi della mesh sono estremamente piccoli o la velocità dell'onda nel materiale è molto alta l'incremento temporale deve essere molto breve. Ma per i problemi di propagazione delle onde ultrasonore questo requisito, in genere molto limitante, non rappresenta un reale inconveniente poiché in queste analisi l'incremento temporale deve rimanere sempre piccolo per garantire l'accuratezza della soluzione. Il principale vantaggio dell'operatore implicito sopra quello esplicito è che, essendo incondizionatamente instabile, non presenta limiti alla dimensione dell'incremento temporale dovuti a requisiti di stabilità, tuttavia i brevi incrementi temporali che i problemi di propagazione di onde richiedono per garantire l'accuratezza della soluzione rendono questo vantaggio inutile facendo si che l'analisi dinamica di tipo implicito risulti molto più onerosa dal punto di vista computazionale di quella esplicita. Il metodo implicito è economicamente attraente solo quando gli 
incrementi temporali possono essere molto più larghi di quelli usati nel caso del metodo esplicito, ma questo non è il caso degli studi riguardanti la propagazione di onde.

Vi sono altri fattori a favore dell'integrazione esplicita. Nei metodi espliciti gli spostamenti sono calcolati utilizzando informazioni relative a spostamenti e derivate rispetto al tempo degli spostamenti noti all'inizio di ogni incremento temporale. Conseguentemente la matrice di massa globale e la matrice di rigidezza non necessitano di essere calcolate e invertite, con grande risparmio computazionale rispetto all'analisi implicita. Inoltre poiché la matrice di rigidezza non necessita di essere formata e memorizzata, il metodo esplicito può lavorare con modelli 3D di grandi dimensioni richiedendo molto meno spazio disco e memoria del metodo implicito per la stessa simulazione. A causa della sua formulazione il metodo esplicito è anche molto più facile da implementare di quello implicito, e può gestire efficientemente non linearità del materiale mentre il metodo implicito in presenza di non linearità significative può avere difficoltà di convergenza [23].

In sintesi, un'analisi dinamica esplicita è molto più efficiente dal punto di vista computazionale per l'analisi di modelli di grandi dimensioni che devono essere studiati per tempo breve, come è il caso dei problemi di propagazione di onda con frequenze nell'ordine dei $\mathrm{MHz}$ che viaggiano in corpi dalle dimensioni non contenute. L'efficienza computazionale della procedura esplicita rispetto a quella implicita per i problemi di propagazione di onde può essere ulteriormente incrementata dall'uso di matrici di massa diagonali.

La simulazione della propagazione delle onde ultrasonore generate tramite laser richiede un'analisi accoppiata termomeccanica. In questo studio si è utilizzata un'analisi termomeccanica accoppiata dove la soluzione meccanica viene ottenuta utilizzando un'integrazione esplicita alle differenze centrate, e le equazioni del trasporto termico sono integrate utilizzando un'integrazione alle differenze finite in avanti.

L'equazione del moto ad un istante specifico è:

$$
[M]\{\ddot{d}\}_{n}+[C]\{\dot{d}\}_{n}+[K]\{d\}_{n}=\{F\}_{n}
$$

dove l'indice $\mathrm{n}$ indica l'incremento temporale, $[\mathrm{M}]$ è la matrice di massa, $[\mathrm{C}]$ è la matrice di smorzamento, $[\mathrm{K}]$ è la matrice di rigidezza, $\{\mathrm{F}\}$ è il vettore delle forze esterne, e $\{\mathrm{d}\}$ è il vettore degli spostamenti. Nel metodo esplicito:

$$
\{d\}_{n+1}=f\left(\{d\}_{n},\{\dot{d}\}_{n},\{\ddot{d}\}_{n},\{d\}_{n-1}, \ldots\right)
$$

Le equazioni del moto del corpo sono integrate utilizzando un'integrazione esplicita alle differenze centrate:

$$
\begin{aligned}
& \{\dot{d}\}_{n}=\frac{1}{2 \Delta t}\left(\{d\}_{n+1}-\{d\}_{n-1}\right) \\
& \{\ddot{d}\}_{n}=\frac{1}{\Delta t^{2}}\left(\{d\}_{n+1}-2\{d\}_{n}+\{d\}_{n-1}\right)
\end{aligned}
$$

dove $\{\dot{d}\}$ è il vettore velocità e $\{\ddot{d}\}$ è il vettore accelerazione. Sostituendo le Eq. (3) e (4) in (1):

$$
\left[\frac{1}{\Delta t^{2}} M+\frac{1}{2 \Delta t} C\right]\{d\}_{n+1}=\{F\}_{n}-[K]\{d\}_{n}+\frac{1}{\Delta t^{2}}[M]\left(2\{d\}_{n}-\{d\}_{n-1}\right)+\frac{1}{2 \Delta t}[C]\{d\}_{n-1}
$$

Se $[M]$ e $[\mathrm{C}]$ sono diagonali allora le equazioni diventano disaccoppiate e gli spostamenti al tempo $\mathrm{n}+1$ possono essere ottenuti senza risolvere le equazioni simultaneamente. La formulazione di tipo "lumped" della matrice di massa determina una matrice di massa diagonale, soddisfacendo il requisito per disaccoppiare le equazioni. Questa formulazione prevede il posizionamento di masse ai nodi di un elemento in modo tale che la somma delle masse dia la massa totale dell'elemento. L'uso di una matrice di massa diagonale è di estrema importanza per l'efficienza computazionale della procedura esplicita. Il vettore delle forze interne $[\mathrm{K}]\{\mathrm{d}\} \mathrm{n}$ può essere calcolato sommando il contributo degli elementi in modo che venga richiesta la formazione della matrice di rigidezza globale. Oltre a disaccoppiare le equazioni, la matrice delle masse diagonale determina un incremento temporale stabile più lungo rispetto ad una formulazione con matrice di massa consistente.

Le equazioni del trasporto termico sono integrate utilizzando un'integrazione alle differenze finite in avanti:

$$
\{T\}_{n+1}=\{T\}_{n}+\Delta t_{n+1}\{\dot{T}\}_{n}
$$

dove $\mathrm{T}$ è la temperatura. 
Siccome sia la soluzione meccanica che quella termica sono esplicite, il trasporto termico e la soluzione meccanica vengono ottenute simultaneamente attraverso un accoppiamento esplicito. Quindi nessuna matrice di rigidezza tangente deve essere calcolata.

Nelle procedure dinamiche esplicite la risoluzione temporale e spaziale delle simulazioni sono di fondamentale importanza per la stabilità e per l'accuratezza della soluzione. Un'approssimazione del limite di stabilità è spesso definita come il più piccolo tempo di transito di un'onda di dilatazione attraverso un qualsiasi elemento della mesh:

$$
\Delta t \approx \frac{L_{\min }}{C_{L}}
$$

dove $L_{\min }$ è la più piccola dimensione di un elemento della mesh e $C_{L}$ è la velocità dell'onda di dilatazione.

Gli ultrasuoni generati tramite laser richiedono degli incrementi temporali molto piccoli per poter risolvere accuratamente le loro componenti ad alta frequenza. Il lavoro di diversi ricercatori [per esempio 11, 19] ha stabilito che una soluzione accurata può essere ottenuta se:

$$
\Delta t=\frac{1}{20 f_{\max }}
$$

dove $f_{\max }$ è la più alta frequenza di interesse.

Una risoluzione spaziale ragionevole per la propagazione di onde può essere ottenuta [11,19] quando la dimensione degli elementi finiti è al massimo 1/10 della più piccola lunghezza d'onda da analizzare.

$$
l_{e}=\frac{\lambda_{\min }}{10}
$$

dove $l_{e}$ è la lunghezza dell'elemento e $\lambda_{\min }$ è la più piccola lunghezza d'onda di interesse.

Come evidenziato nell'introduzione un beneficio fondamentale nell'utilizzare il laser per generare onde acustiche consiste nella possibilità di effettuare ispezioni remote, che non dipendono dalle condizioni di contatto e consentono la realizzazioni di sistemi interamente automatizzati. Tuttavia affinché l'ispezione possa essere totalmente remota anche il ricevitore deve lavorare senza contatto. Se un trasduttore non a contatto viene impiegato in ricezione allora per simulare il setup sperimentale completo bisogna modellare anche la propagazione di onde in aria. Il grado di libertà dei nodi degli elementi finiti utilizzati per discretizzare l'aria è la pressione acustica, che rappresenta la deviazione della pressione locale dalla pressione ambiente causata dell'onda sonora. Il modello agli EF della propagazione di onde ultraonore attraverso l'aria richiede un costo computazionale notevole perché la velocità del suono in aria è di $343 \mathrm{~m} / \mathrm{s}$, che rappresenta un valore molto più basso della velocità di un'onda longitudinale in un solido, per esempio per l'acciaio questo valore è 5990 $\mathrm{m} / \mathrm{s}$. Siccome la frequenza delle onde ultrasonore generate in un solido rimane immutata quando sono trasmesse dalla superficie in vibrazione del solido all'aria, la lunghezza d'onda deve ridursi in modo significativo, come può evincersi dalla relazione:

$$
C_{s s}=\lambda_{\text {air }} f
$$

dove $C_{s s}$ è la velocità del suono in aria $343 \mathrm{~m} / \mathrm{s}$, $\lambda_{\text {air }}$ è la lunghezza d'onda in aria, e $f$ è la frequenza che per le onde ultrasonore generate tramite laser è nel range dei $\mathrm{MHz}$.

Per esempio se un'onda è stata generata in un solido di acciaio, il rapporto tra la lunghezza d'onda nell'acciaio e la lunghezza d'onda in aria sarà:

$$
\frac{C_{L}}{C_{s s}}=\frac{\lambda_{\text {steel }} f}{\lambda_{\text {air }} f} \Rightarrow \frac{\lambda_{\text {steel }}}{\lambda_{\text {air }}}=\frac{C_{L}}{C_{s s}}=\frac{5990}{343}=17.463 \Rightarrow \lambda_{\text {air }}=\frac{\lambda_{\text {steel }}}{17.463}
$$

In base all'equazione (9), una risoluzione spaziale delle onde che si propagano attraverso l'aria richiederà una dimensione degli elementi finiti circa 17.5 volte più piccola rispetto alla dimensione degli elementi finiti utilizzati per modellare l'acciaio. Nel caso di un'onda di frequenza $\mathrm{f}=1 \mathrm{MHz}$ l'Eq. (9) richiederebbe:

$$
l_{e}^{\text {air }}=\frac{\lambda_{\text {air }}}{10}=\frac{C_{s s}}{10 \cdot f}=\frac{343 \mathrm{~m} / \mathrm{s}}{10 \cdot\left(1 \cdot 10^{6} 1 / \mathrm{s}\right)}=34.3 \cdot 10^{-6} \mathrm{~m}=0.0343 \mathrm{~mm}
$$


dove $l_{e}^{a i r}$ è la dimensione richiesta per la lunghezza dell'elemento nella porzione di aria da studiare. Questo risultato dimostra chiaramente come sia importante limitare la quantità d'aria da considerare nell'analisi; altrimenti il costo computazionale diventerà spesso insostenibile. Un modo di risolvere il problema è di implementare condizioni al contorno di non riflessione (non-reflecting boundary conditions), che sono utilizzate per problemi di strutture in vibrazione in un mezzo acustico di dimensioni infinite. Le condizioni al contorno di non riflessione consentono alle onde di uscire dal dominio senza riflessioni. Quindi, a meno che in prossimità del trasduttore ci siano altri solidi dove le onde acustiche potrebbero essere riflesse, le condizioni al contorno di non riflessione consentono di modellare solo la porzione di aria fra il trasduttore non a contatto e il solido che si sta testando. Le condizioni al contorno di non riflessione che sono state implementate nel modello numerico sono in grado di evitare qualsiasi riflessione per ogni angolo di incidenza possibile.

\section{RISULTATI}

$\mathrm{L}$ 'uso di ultrasuoni generati tramite laser come strumento per l'individuazione di difetti è stato esteso recentemente anche alle rotaie. In questo studio si è focalizzata l'attenzione sulla efficienza della tecnica numerica presentata nel simulare la propagazione di onde ultrasonore generate tramite laser e con frequenze nell'ordine dei $\mathrm{MHz}$. Sia le analisi numeriche che sperimentali sono state condotte sulla rotaia del tipo $136 \mathrm{lb}$ AREMA. Diversi casi sono stati considerati; qui presentiamo i risultati che riproducono i test non distruttivi del fungo della rotaia, con e senza difetti.

Inizialmente l'approccio numerico è stato convalidato tramite confronto con risultati analitici relativi ad onde guidate che si propagano in un anello circolare.

\section{Confronto con una solurione analitica - Anello circolare}

Per convalidare l'approccio proposto, l'accuratezza della simulazione numerica è verificata tramite confronto con una soluzione analitica $[11,24]$. Liu e Qu hanno sviluppato un metodo generale di risoluzione dei problemi di propagazione dinamica di onde in un anello circolare soggetto ad una trazione superficiale che varia nel tempo [24].

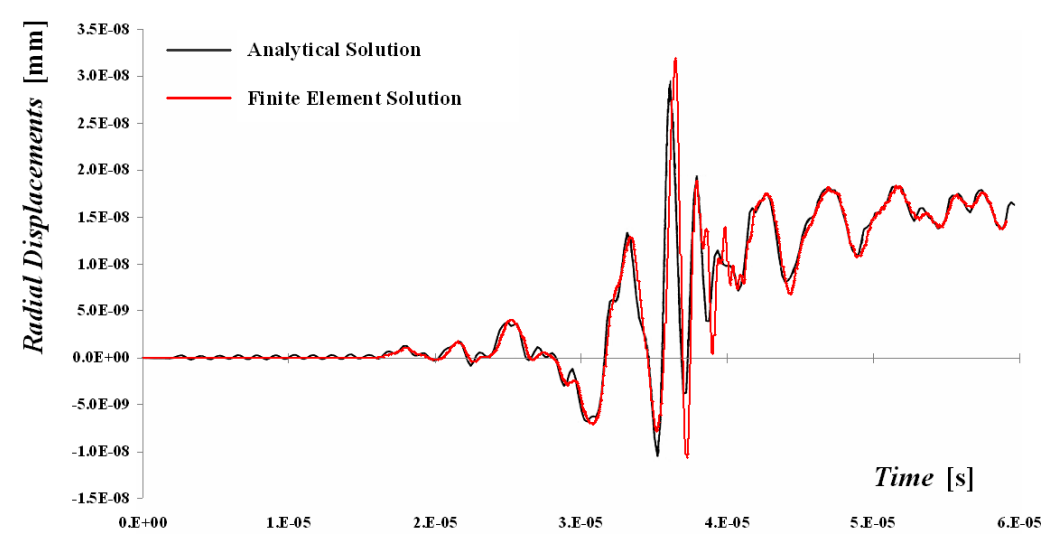

Figura 1: Confronto tra la soluzione analitica e quella agli elementi finiti.

Gli autori hanno assunto che la superficie interna dell'anello fosse scarica da tensioni mentre quella esterna è sollecitata tramite un carico variabile nel tempo. Il metodo utilizzato è quello dell' eigenfunction expansion. La risposta nel tempo dell'anello è ottenuta tramite sovrapposizione di tutte le eigenfunctions per tutte le possibile frequenze. Moser et al. [11] hanno utilizzato l'approccio sviluppato da Liu e Qu [24] per verificare la validità del loro approccio numerico basato sul metodo degli elementi finiti con integrazione implicita. La struttura dell'anello considerata viene mostrata in [11, Fig. 5], e le sue proprietà (acciaio) sono sintetizzate in [11, Tab. 1] come Material II. L'anello è caricato sulla superficie esterna con una forza puntuale, $f(t)$, che agisce perpendicolarmente alla superficie. La funzione del carico, $f(t)$, e il suo contenuto in frequenza sono dati in [11, Fig. 6].

Gli spostamenti radiali, $u_{r}$, calcolati tramite la soluzione analitica per un punto che si trova ad un angolo di $90^{\circ}$ tra la sorgente e il ricevitore sono plottati nella Fig. 1 e confrontati con i risultati ottenuti tramite l'approccio presentato in questo lavoro. Le soluzioni risultano molto vicine, dimostrando che il metodo proposto è molto accurato nel simulare problemi di propagazione di onde. 


\section{Studio sul coefficiente di assorbimento e sul diametro dell'areola d'impatto}

Per calcolare esattamente l'entità del flusso di calore da utilizzare nelle analisi numeriche, per la modellazione del fenomeno, a partire dall'energia totale proveniente dal laser, è stato eseguito uno studio sperimentale per ricavare il coefficiente di assorbimento della superficie del provino di acciaio. Si è mantenuta costante l'energia e diminuita progressivamente l'area d'impatto in modo da aumentare la densità di potenza incidente. Si è osservato che la risposta è rimasta della tipologia tipica di un regime termoelastico fino ad una densità pari a circa $48.5 \mathrm{MW} / \mathrm{cm}^{2}$ che è nettamente superiore al limite teorico calcolato per il materiale del provino $\left(31 \mathrm{MW} / \mathrm{cm}^{2}\right)$. Ciò significa che la radiazione incidente sul provino viene in parte assorbita ed in parte riflessa, e poiché $31 \mathrm{MW} / \mathrm{cm}^{2}$ è circa il $64 \%$ di $48.5 \mathrm{MW} / \mathrm{cm}^{2}$ si può dichiarare che il coefficiente di assorbimento della superficie metallica del provino è pari a $\alpha=0.64$, mentre il coefficiente di riflessione equivale a $\varrho=0.36$. Si è preferito ricavare sperimentalmente il coefficiente di riflessione, piuttosto che affidarsi a qualche valore, reperibile nelle tabelle, perché esso varia notevolmente con la tipologia di finitura superficiale e con la lunghezza d'onda della radiazione (nel nostro caso $1064 \mathrm{~nm}$ ). Nella tabella seguente sono riportate le prove eseguite per la determinazione del coefficiente $\alpha$ e sono rappresentati i segnali acquisiti nella sezione di sparo.

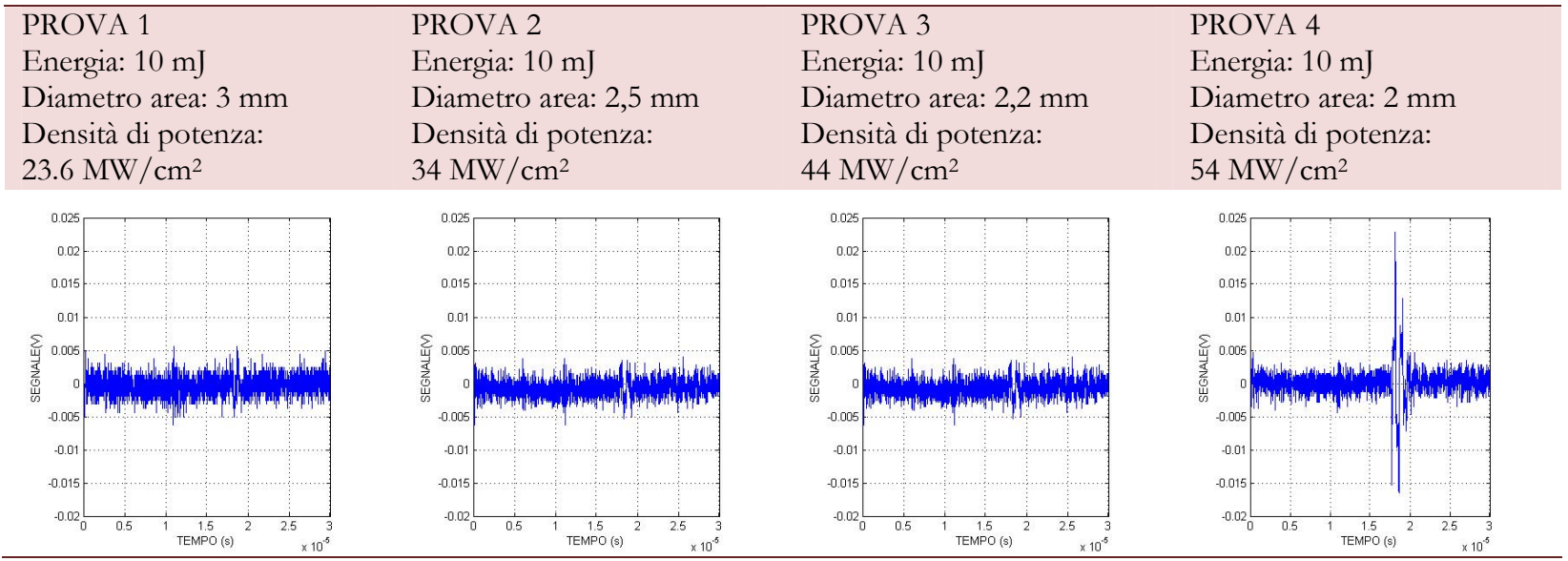

Tabella 1: Prove sperimentali per ricavare il coefficiente di riflessione della superficie metallica.

Il coefficiente di riflessione trovato, comunque, non è molto diverso da quello che si può calcolare tramite una nota espressione analitica [3]:

$$
R=\frac{2-2 \varepsilon+\varepsilon^{2}}{2+2 \varepsilon+\varepsilon^{2}}
$$

dove $\varepsilon=\mu_{0} \sigma c \delta$ essendo $c=300000 \mathrm{Km} / \mathrm{s}$ la velocità della luce nel vuoto, $\mu_{0}=4 \pi 10^{-7} \mathrm{Hm}^{-1}$ la permeabilità magnetica nel vuoto, $\sigma$ la conducibilità elettrica e $\delta$ lo skin depth. Quest'ultimo si può ricavare tramite la seguente espressione [3]:

$$
\delta=\left(\pi \sigma \mu_{r} \mu_{0} \nu\right)^{-\frac{1}{2}}
$$

dove $\mu_{r}$ è la permeabilità magnetica relativa e $v=2.8210^{14} \mathrm{~Hz}$ è la frequenza della radiazione (assumendo $\lambda=1064 \mathrm{~nm}$ ).

Sostituendo i seguenti valori riguardanti un acciaio da costruzione al carbonio, $\mu_{r}=1900$ e $\sigma=7.0410^{6}(\Omega \mathrm{m})^{-1}$, otteniamo $\delta$ $=0.258 \mathrm{~nm}$ e $\varepsilon=0.68$ e quindi $R=0.30$.

Poiché $\varrho$ risulta essere prossimo al valore calcolato analiticamente, $R$, si conclude che il valore sperimentale della riflessività approssima bene il valore reale, con un errore dell'ordine di qualche punto percentuale.

In Fig. 2 e 3 è riportato il setup sperimentale per effettuare le prove a diametro dell'areola variabile. Si è visto che aumentando il diametro della sorgente termoelastica, a parità di energia del fascio laser, si evidenzia uno spostamento del massimo della curva dell'ampiezza dell'onda longitudinale in funzione dell'angolo di ricezione, come rappresentato in Fig. 4.

Altre prove numeriche e sperimentali, che verranno presentate in un successivo articolo, hanno permesso di determinare che il fenomeno della regressione del massimo dell'ampiezza del segnale relativo all'onda longitudinale verso valori minori dell'angolo di ricezione è dovuto alla distribuzione gaussiana dell'energia sulla superficie d'incidenza. Tale fenomeno può essere trascurato per areole molto piccole ma non per aree circolari di diametro maggiore di $3 \mathrm{~mm}$. 


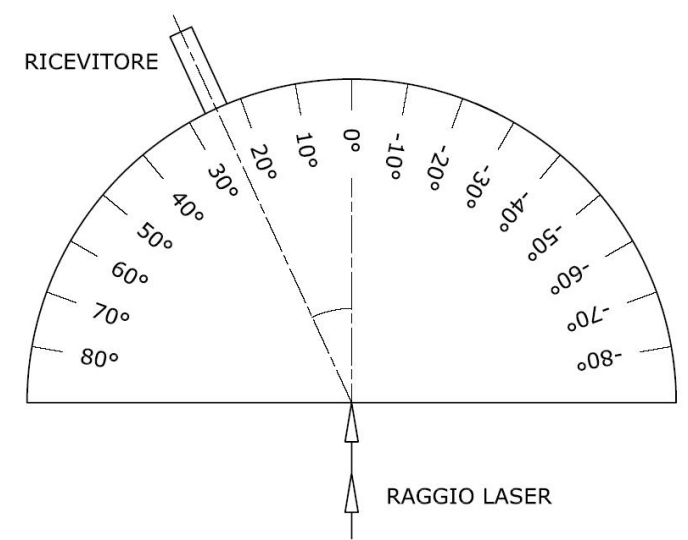

Figura 2: Disegno schematico del setup sperimentale.

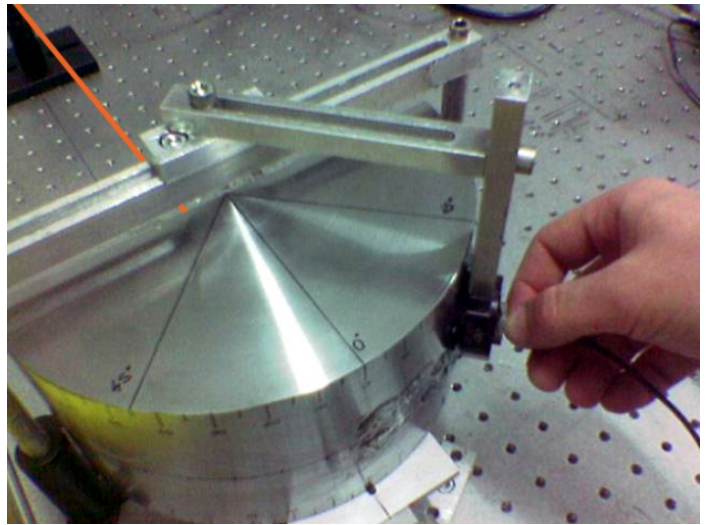

Figura 3: Foto del provino e del sensore per le prove a direzione di ricezione variabile. La linea arancione è tracciata per rappresentare il raggio laser.

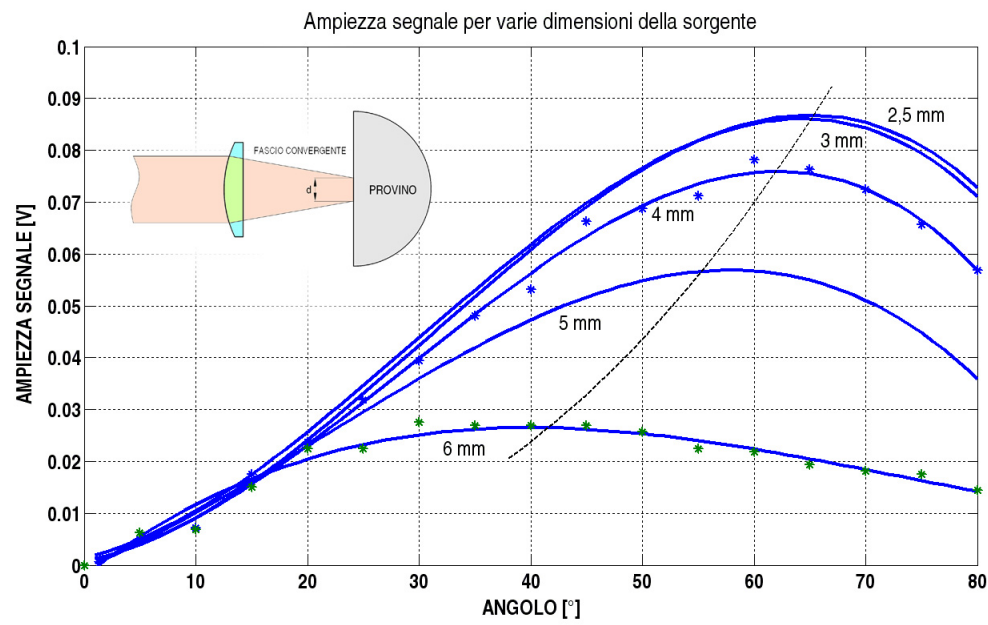

Figura 4: Ampiezza del segnale relativo all'onda longitudinale al variare dell'angolo di ricezione per diversi diametri dell'area irradiata e un'energia di $10 \mathrm{~mJ}$. La curva tratteggiata interpola i massimi delle varie curve.

Altre prove numeriche e sperimentali, che verranno presentate in un successivo articolo, hanno permesso di determinare che il fenomeno della regressione del massimo dell'ampiezza del segnale relativo all'onda longitudinale verso valori minori dell'angolo di ricezione è dovuto alla distribuzione gaussiana dell'energia sulla superficie d'incidenza. Tale fenomeno può essere trascurato per areole molto piccole ma non per aree circolari di diametro maggiore di $3 \mathrm{~mm}$.

\section{Test del fungo senza difetti}

Il primo setup sperimentale è mostrato nelle Fig. 5 e 6 a). Il fascio laser è focalizzato in un punto di $1 \mathrm{~mm}$ di diametro sul lato del fungo della rotaia (tipo $136 \mathrm{lb}$ AREMA). Il laser ha un'energia massima di $0.75 \mathrm{~J}$, con una durata dell'impulso di $6 \cdot 10^{-9}$ secondi. L'area colpita dallo sparo laser viene riscaldata dall'energia ottica che genera le onde ultrasonore per l'effetto termoelastico.

Nella simulazione numerica, il modello della rotaia è stato discretizzato per essere pienamente corrispondente alla configurazione sperimentale e misurare gli spostamenti e le tensioni generati dall'onda ultrasonora. Nei test sperimentali è stato usato un trasduttore piezoelettrico, con un'area sensibile di $12.7 \mathrm{~mm}$ di diametro e frequenza nominale di $1 \mathrm{MHz}$, per registrare un segnale in Volt, proporzionale allo spostamento, sulla superficie del fungo della rotaia, come mostrato in Fig. 6a). La rotaia del tipo $136 \mathrm{lb}$ AREMA è in acciaio con alcune percentuali di Mn, P, S, e Si; altri elementi che generalmente vengono aggiunti per migliorare le proprietà meccaniche sono Ni, Cr, Mo, V [25]. Nello studio numerico vengono assunte le seguenti proprietà per il materiale della rotaia: densità $7800 \mathrm{Kg} / \mathrm{m}^{3}$, modulo di Young $207 \cdot 10^{9} \mathrm{~Pa}$, coefficiente di Poisson 0.3, coefficiente di espansione termica $1.3 \cdot 10^{-5} \mathrm{~K}^{-1}$, calore specifico $490 \mathrm{~J} \cdot \mathrm{Kg}^{-1} \cdot \mathrm{K}^{-1}$, e conduttività termica $46 \mathrm{~W} \cdot \mathrm{m}^{-1} \cdot \mathrm{K}^{-1}$. 


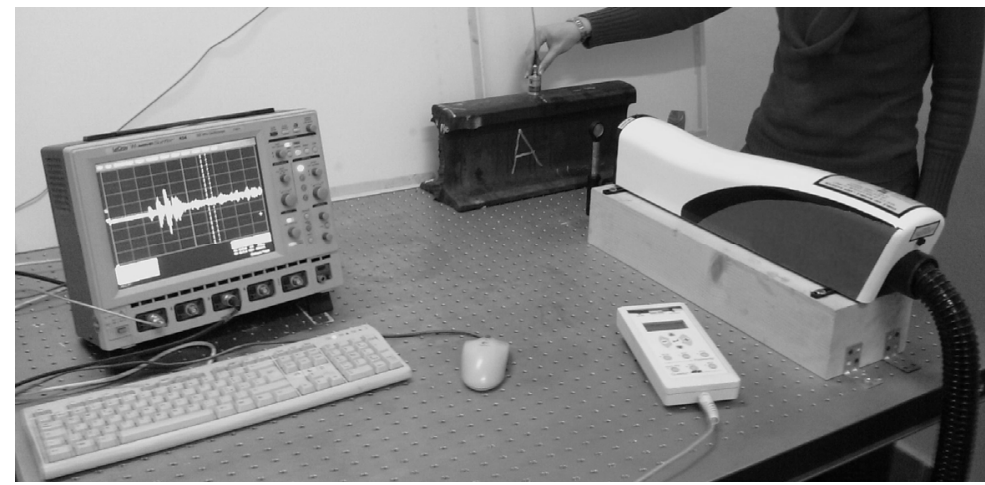

Figura 5: Foto del setup sperimentale per lo studio del fungo della rotaia.

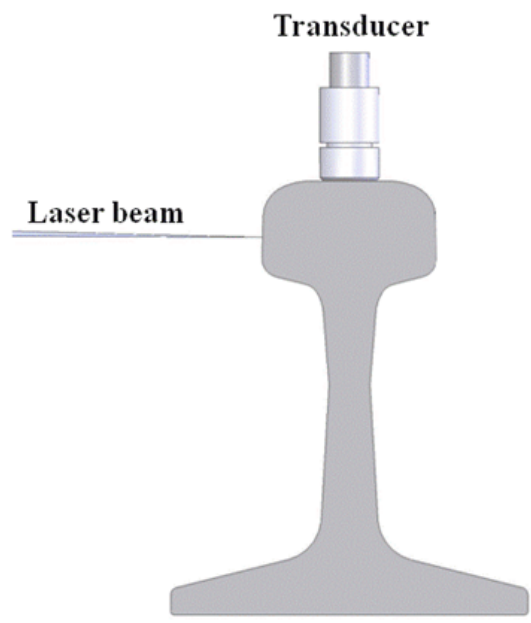

(a)

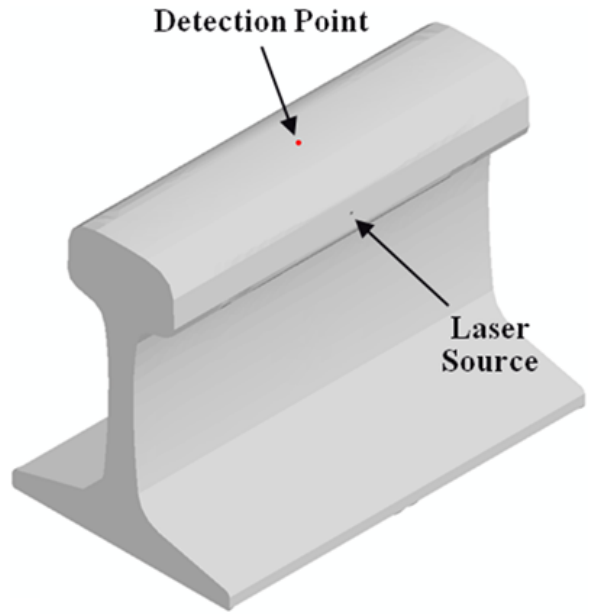

(b)

Figura 6: a) Schema del setup sperimentale e b) modello agli elementi finiti della rotaia senza difetti.

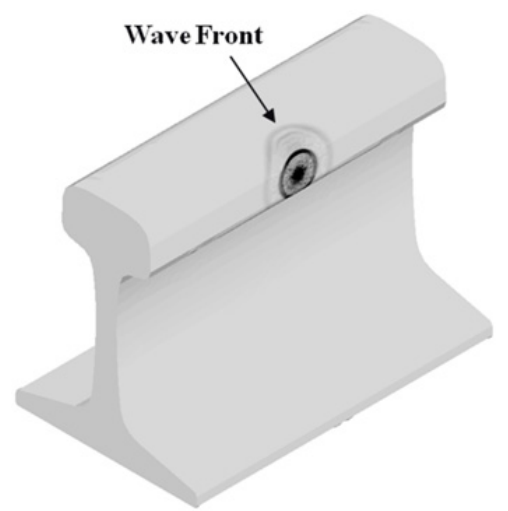

Figura 7: Mappa degli spostamenti dall'approccio agli elementi finiti. Il fronte d'onda è indicato; le regioni nere indicano spostamenti che eccedono il valore di $1 \cdot 10^{-4} \mathrm{~mm}$

Utilizzando la formulazione numerica descritta nel precedente paragrafo, le onde ultrasonore generate in regime termoelastico sono calcolate nel modello agli elementi finiti che riproduce in dettaglio la geometria della rotaia, Fig. 6b. La Fig. 7 mostra la mappa degli spostamenti, determinata tramite il metodo degli elementi finiti, che non superano il valore di $1 \cdot 10^{-4} \mathrm{~mm}$, permettendo di visualizzare chiaramente il fronte d'onda dopo $5 \cdot 10^{-6}$ secondi dallo sparo laser. Il limite nella mappa degli spostamenti è necessario poiché gli spostamenti sono molto alti nel punto dove il fascio laser colpisce la rotaia, senza questo limite tutte le diverse tonalità di grigio destinate ad indicare i diversi range di spostamento sarebbero localizzate nel punto dove il laser colpisce la rotaia mentre tutte le altre aree sarebbero rappresentate in unica tonalità di grigio. 
Il grafico in Fig. 8a mostra il segnale acquisito dal sensore piezoelettrico posizionato sul fungo della rotaia. La Fig. 8b presenta gli spostamenti normali alla superficie misurati tramite l'approccio numerico nello stesso punto. Esaminando il tempo di arrivo delle diverse onde mostrate nelle Fig. $8 \mathrm{a}$ e b, si vede che la prima onda corrisponde al tempo teorico di arrivo dell'onda longitudinale, $\mathrm{C}_{1}$, che propaga direttamente dalla sorgente al ricevitore. La distanza dalla sorgente laser al ricevitore è circa $43 \mathrm{~mm}$; il tempo di arrivo teorico, che corrisponde sia al valore numerico che sperimentale, è $\mathrm{t}_{1}=43 / 5.99=7.2 \mu \mathrm{s}$. Il tempo di arrivo teorico per l'onda di taglio che viaggia direttamente dalla sorgente al ricevitore, $\mathrm{C}_{2}$, deve essere $\mathrm{t}_{2}=43 / 3.2=13.4 \mu \mathrm{s}$, sapendo che la velocità dell'onda di taglio nell'acciaio è $3.2 \mathrm{~mm} \cdot \mu \mathrm{s}^{-1}$. Infine l'onda di Rayleigh, $C_{R}$, che si propaga lungo la superficie della rotaia per una distanza di circa $53.5 \mathrm{~mm}$ ad una velocità di 2.97 $\mathrm{mm} \cdot \mu \mathrm{s}^{-1}$, arriva al ricevitore dopo circa $18 \mu \mathrm{s}$. La propagazione dell'onda di Rayleigh può essere vista chiaramente nella simulazione numerica; qui non è mostrata per brevità. Esperimenti e simulazioni mostrano risultati molto simili, provando l'efficienza del metodo nel simulare la generazione degli ultrasuoni con il laser, per frequenze nel range dei MHz.
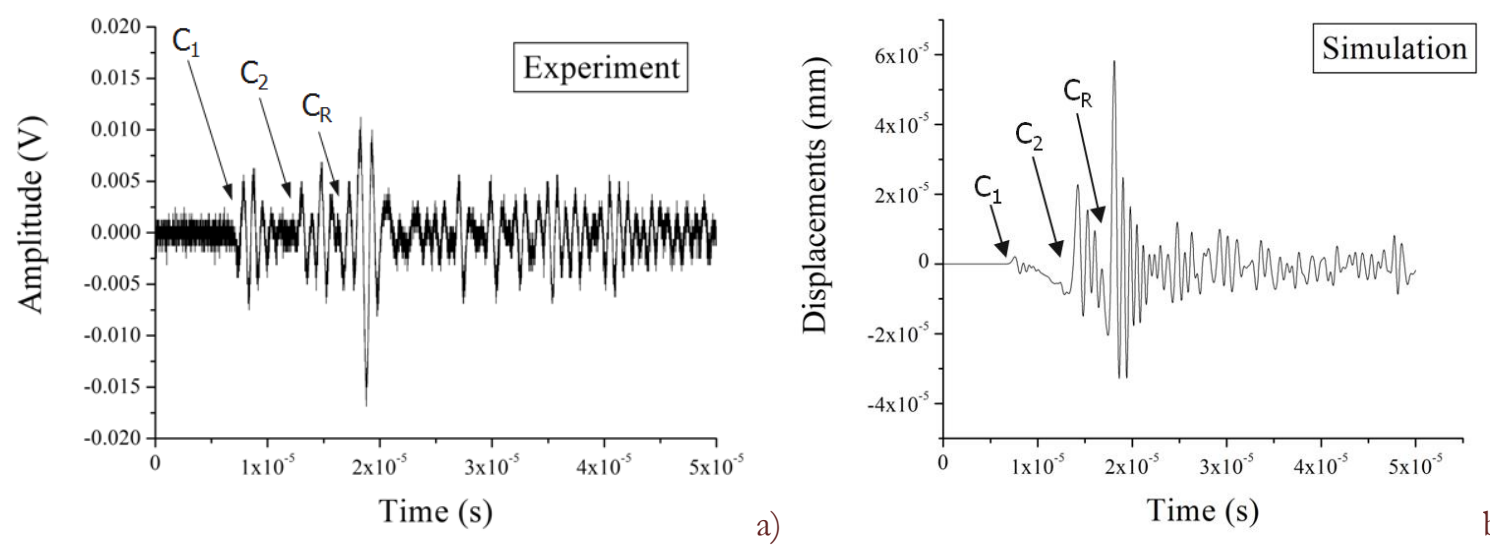

b)

Figura 8: a) Tensione elettrica, proporzionale agli spostamenti superficiali, in funzione del tempo per il trasduttore posizionato sopra il fungo; b) spostamenti normali alla superficie misurati con l'approccio numerico nello stesso punto.

\section{Test del fungo con difetti}

Il terzo setup sperimentale è mostrato in Fig. 9a. La configurazione è analoga al caso precedente ma in questo caso il fungo della rotaia ha un difetto noto come spaccatura verticale (VSH), mostrato in Fig. 10. Questo difetto si estende verticalmente nel fungo ma propaga nella direzione longitudinale della rotaia.
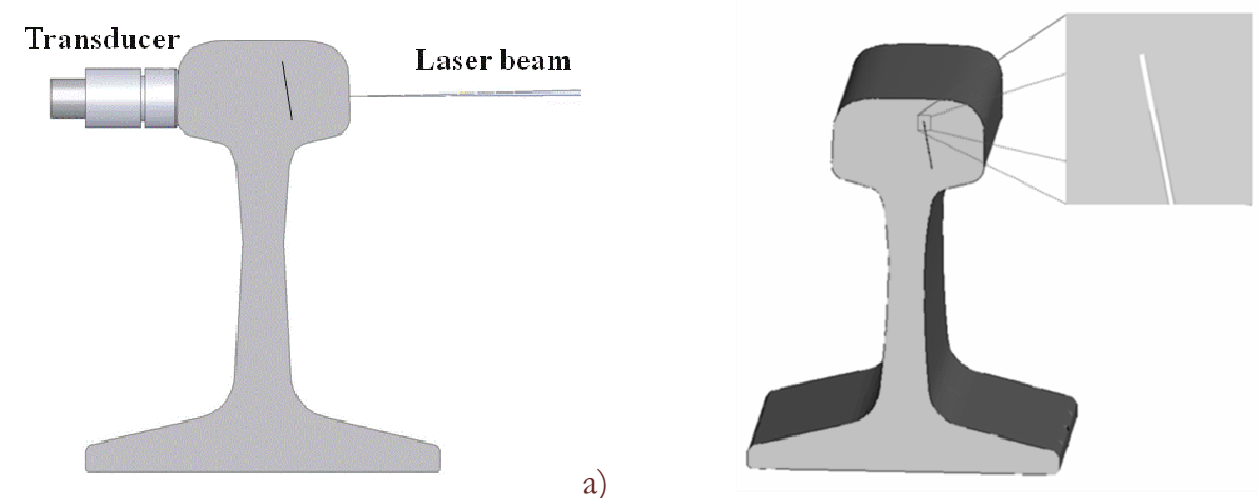

Figura 9: a) Schema del setup sperimentale e b) modello agli elementi finiti della rotaia con difetto nel fungo.

Nella simulazione numerica il modello della rotaia è fatto in modo da riprodurre perfettamente la configurazione sperimentale, ma la geometria della spaccatura verticale nel fungo non è nota. Cosi nel modello agli elementi finiti è stato introdotto un difetto che riproduce approssimativamente il difetto visibile nella sezione finale della rotaia, anche se tale sezione è distante dal punto in cui il laser colpisce la rotaia. Non conoscendo la geometria esatta del difetto e la posizione nel fungo non ci si aspetta una perfetta corrispondenza tra i risultati numerici e sperimentali. Viene quindi studiato come 
la presenza del difetto nel modello agli elementi finiti può influenzare le onde ultrasonore che arrivano nel punto dove il sensore è posizionato. I risultati numerici devono qualitativamente corrispondere agli esperimenti, dove è stato trovato che la presenza del difetto attenua visibilmente il segnale iniziale.

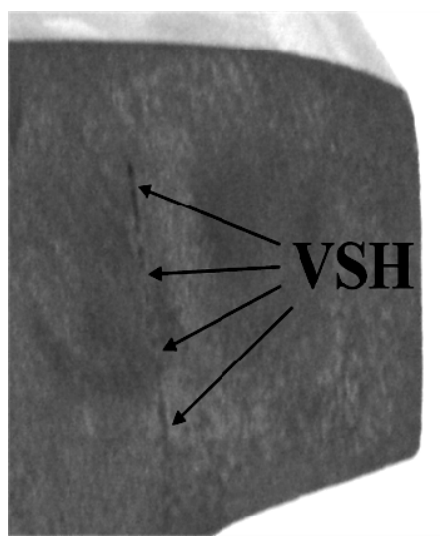

Figura 10: Difetto nel fungo noto come spaccatura verticale (VSH).

Nella Fig. 11 vengono confrontati i risultati per il fungo con e senza difetto. Nella rotaia senza difetto la prima onda arriva dopo circa $11.5 \mu$ s e i valori di spostamento nell'intervallo temporale da $12 \mu$ s a $15 \mu$ s sono nel range di $10^{-6} \mathrm{~mm}$. Nella rotaia con difetto la prima onda arriva dopo circa $12.5 \mu$ s e i valori di spostamento nell'intervallo di tempo da $12.5 \mu$ s a 15 $\mu$ s sono nel range di $10^{-7} \mathrm{~mm}$, molto attenuati rispetto al valore determinato nella rotaia senza difetto. Questo risultato è qualitativamente in buon accordo con gli esperimenti.

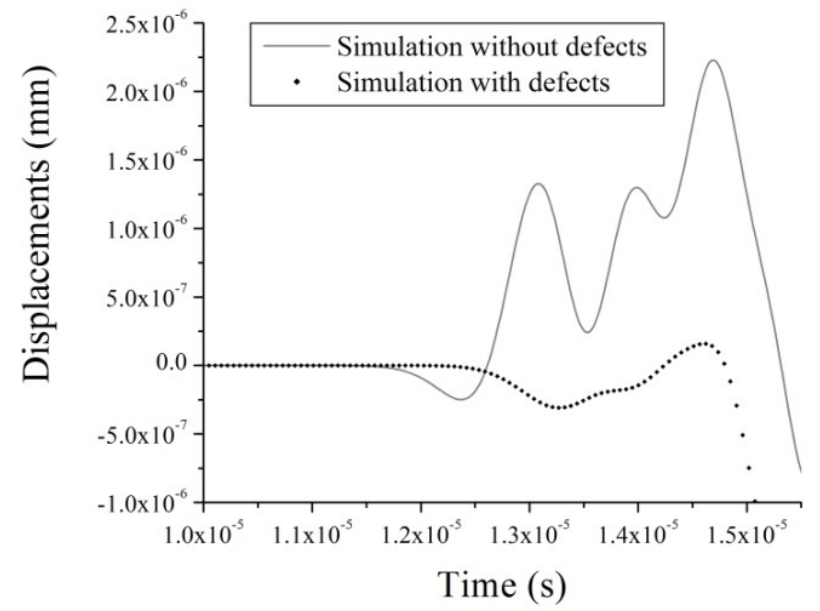

Figura 11: Spostamenti normali alla superficie misurati con l'approccio numerico sul lato opposto del fungo rispetto allo sparo laser. Risultati a confronto per il fungo con e senza difetto.

La Fig. 12 mostra la mappa degli spostamenti del fungo con difetto in quattro istanti differenti, da $2 \mu$ s a $8 \mu$ s, determinati numericamente. Le regioni nere hanno spostamenti che eccedono il valore di $1 \cdot 10^{-9} \mathrm{~mm}$, al fine di identificare il fronte d'onda. Le onde non attraversano il difetto, cosi quando il fronte d'onda raggiunge la discontinuità nel materiale si propaga attorno ad essa. Questo causa un ritardo nell'arrivo dell'onda ed anche una attenuazione del campo degli spostamenti iniziali, nel punto dove sono rilevati gli spostamenti.

Nella Fig. 13 il limite nella mappa degli spostamenti è stato portato a $1 \cdot 10^{-5} \mathrm{~mm}$ al fine di visualizzare le principali caratteristiche della propagazione dell'onda nel fungo con difetto. I risultati numerici sono mostrati nella sezione centrale della rotaia, dove il fascio laser colpisce la rotaia, in vista $2 \mathrm{D}$ e $3 \mathrm{D}$, dove metà del modello è stato rimosso per visualizzare la sezione centrale. Le mappe degli spostamenti mostrate in Fig. 13 sono state determinate dopo 18$\mu$ s dallo sparo laser; in questo istante l'onda di Rayleigh, che non è influenzata dalla presenza del difetto, ha percorso lungo la superficie della 
rotaia una distanza di circa $53.5 \mathrm{~mm}$ dal punto di impatto del laser al top della rotaia alla velocità di $2.97 \mathrm{~mm} / \mu \mathrm{s}$. Questo è il motivo per cui troviamo l'onda di Rayleigh nel punto indicato in Fig. 13.
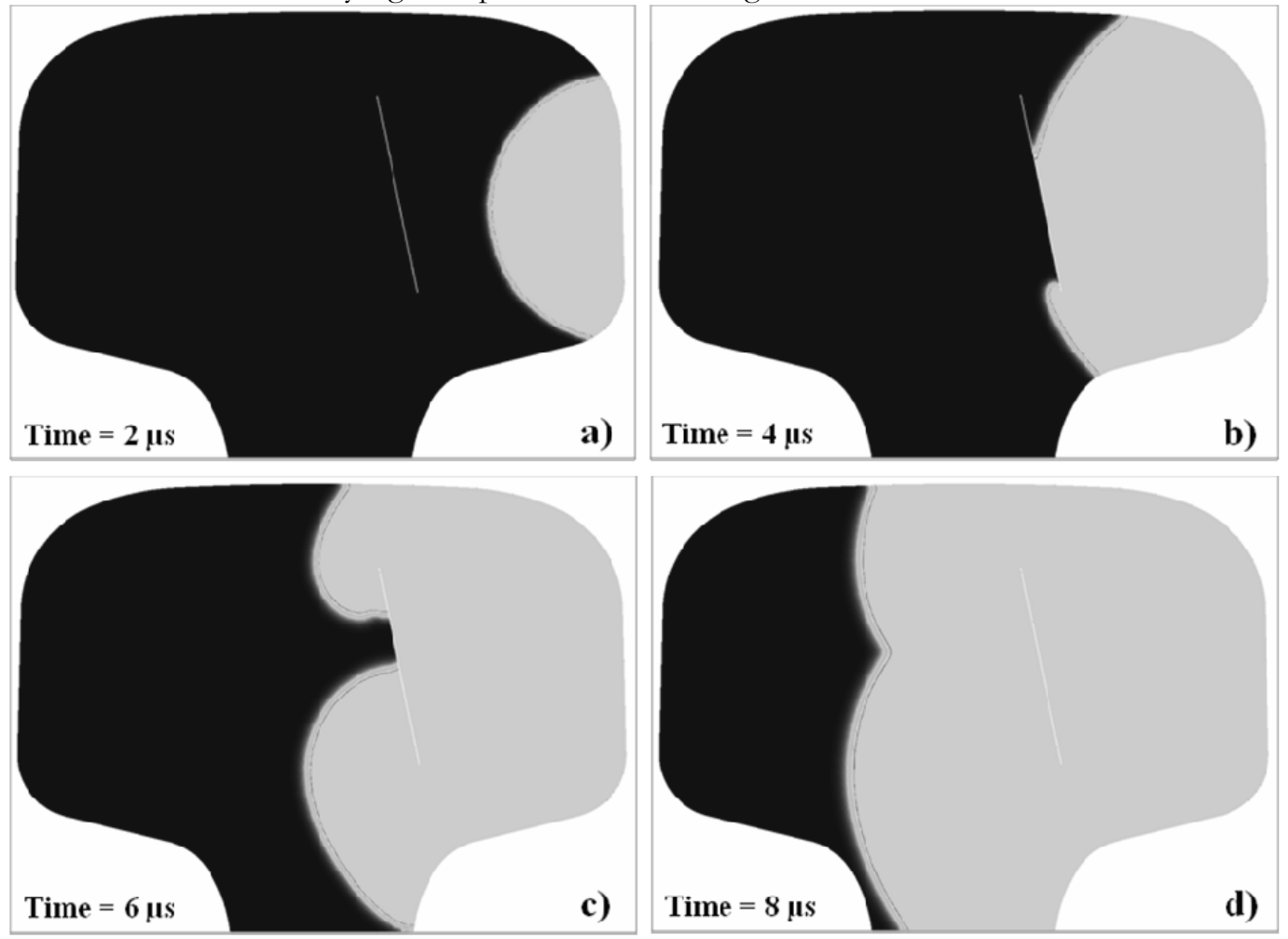

Figura 12: Mappa degli spostamenti in quattro istanti differenti dal modello agli elementi finiti per lo studio del fungo senza difetti. Le regioni nere hanno spostamenti che eccedono il valore di $1 \cdot 10^{-9} \mathrm{~mm}$ al fine di visualizzare chiaramente il fronte d'onda.
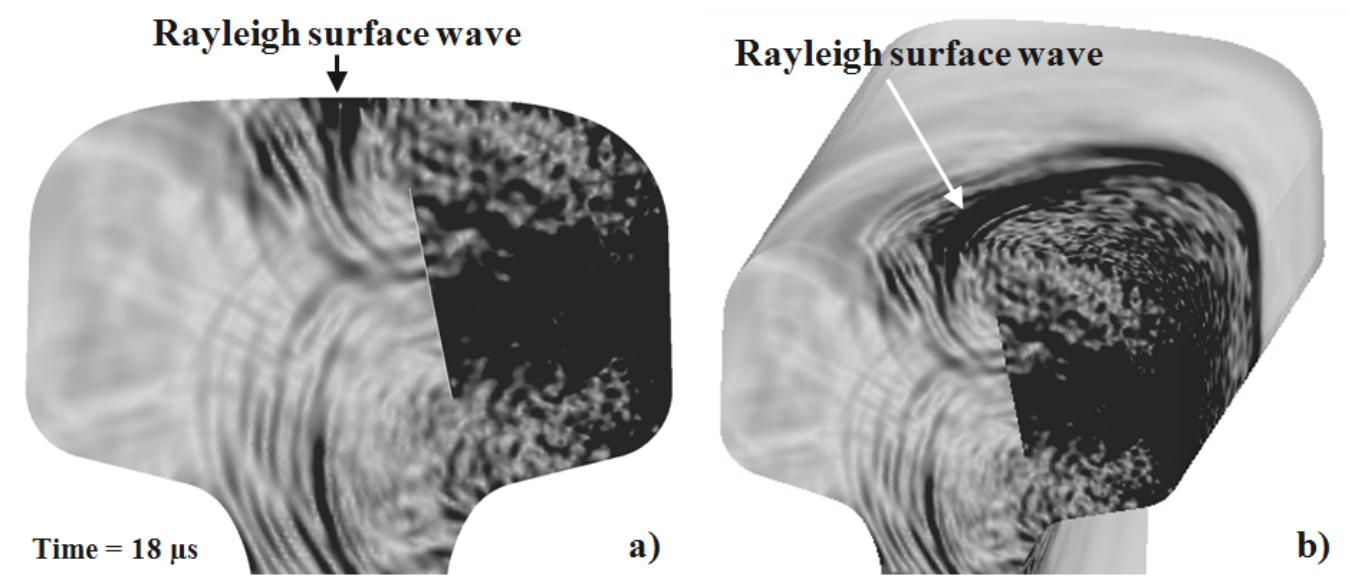

Figura 13: Mappa degli spostamenti al tempo $18 \mu$ s dal modello agli elementi finiti per il fungo. Immagine 2D, a), e 3D, b), dove metà del modello è stato rimosso. Le regioni nere hanno spostamenti che eccedono il valore di $1 \cdot 10^{-5} \mathrm{~mm}$.

Test del fungo con trasduttore senza contatto

Il primo setup sperimentale è mostrato nelle Fig. 14 e 15 a). Il fascio laser è focalizzato in un punto centrale del lato del fungo su un'area di $1 \mathrm{~mm}$ di diametro. Nella simulazione numerica il modello della rotaia, Fig. $15 \mathrm{~b}$ ), è stato discretizzato in modo da ottenere un corrispondenza con la configurazione sperimentale. Nei test è stato impiegato un trasduttore senza contatto con la rotaia, per ricevere le onde dopo aver attraversato l'aria. Il trasduttore ha una frequenza nominale di $1 \mathrm{MHz}$ e registra un segnale proporzionale alla pressione acustica, che è la deviazione locale della pressione rispetto a quella ambiente causata dall'onda ultrasonora. La distanza tra la superficie della rotaia e il trasduttore è $10 \mathrm{~mm}$. Nel 
modello numerico le proprietà dell'aria sono state prese come segue: modulo di comprimibilità $1.42 \cdot 10^{5} \mathrm{~Pa}$ e densità 1.2 $\mathrm{kg} / \mathrm{m}^{3}$.

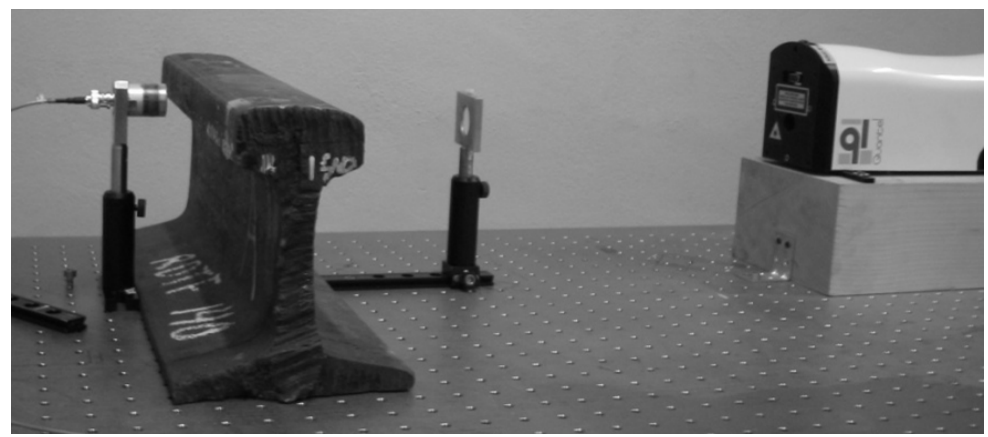

Figura 14: Foto del setup sperimentale per lo studio del fungo.

Le onde ultrasonore generate in regime termoelastico attraversano il fungo e arrivano sul lato opposto rispetto alla sorgente dove è posizionato il trasduttore. Le onde ultrasonore attraversano quindi l'aria, per una distanza di $10 \mathrm{~mm}$, prima di raggiungere il trasduttore che rileverà la variazione di pressione sulla sua superficie. Le Fig. 16 da a) ad i) plottano le mappe della pressione acustica, che è la deviazione della pressione locale dalla pressione ambiente causata da un'onda ultrasonora, nella sezione della porzione di aria tra la superficie della rotaia e il trasduttore non a contatto in nove istanti differenti. La sezione è normale all'asse della rotaia e passa per il centro di massa della porzione di aria. Come già visto in [26, Fig. 14] per la rotaia senza difetto, le onde ultrasonore viaggiano attraverso il fungo in $\mathrm{t}_{1}=11.4 \mu \mathrm{s}$, quindi attraversano l'aria alla velocità di $343 \mathrm{~m} / \mathrm{s}$. Poiché la distanza in aria è di $10 \mathrm{~mm}$, l'onda impiegherà $\mathrm{t}_{2}=10 / 343000=29.1 \mu \mathrm{s}$ per raggiungere il trasduttore; cosi il tempo totale dallo sparo laser è $\mathrm{t}_{1}+\mathrm{t}_{2}=40 \mu \mathrm{s}$. La Fig. 16a mostra le onde ultrasonore che viaggiano in aria dopo $20 \cdot 10^{-6}$ secondi dallo sparo laser. Come determinato, dopo circa $40 \mu$ s l'onda raggiunge il trasduttore, ciò è mostrato in Fig. 16c ed anche in Fig. $16 \mathrm{k}$ dove è stato imposto un limite di $1 \cdot 10^{-8} \mathrm{~N} / \mathrm{mm}^{2} \mathrm{nella}$ mappa della massima pressione acustica per evidenziare il fronte d'onda che arriva al tempo teorico, non chiaramente visibile in Fig. 16c. Nelle Fig. 16d, 16e, 16f si possono notare diverse onde che arrivano al trasduttore con una pressione acustica elevata; questo è confermato dall'analisi dei risultati sperimentali ottenuti nell'intervallo da $55 \cdot 10^{-6}$ a $70 \cdot 10^{-6}$ secondi. Le Fig. 16 da g) a i) mostrano la mappa di pressione acustica per la parte finale dell'analisi.
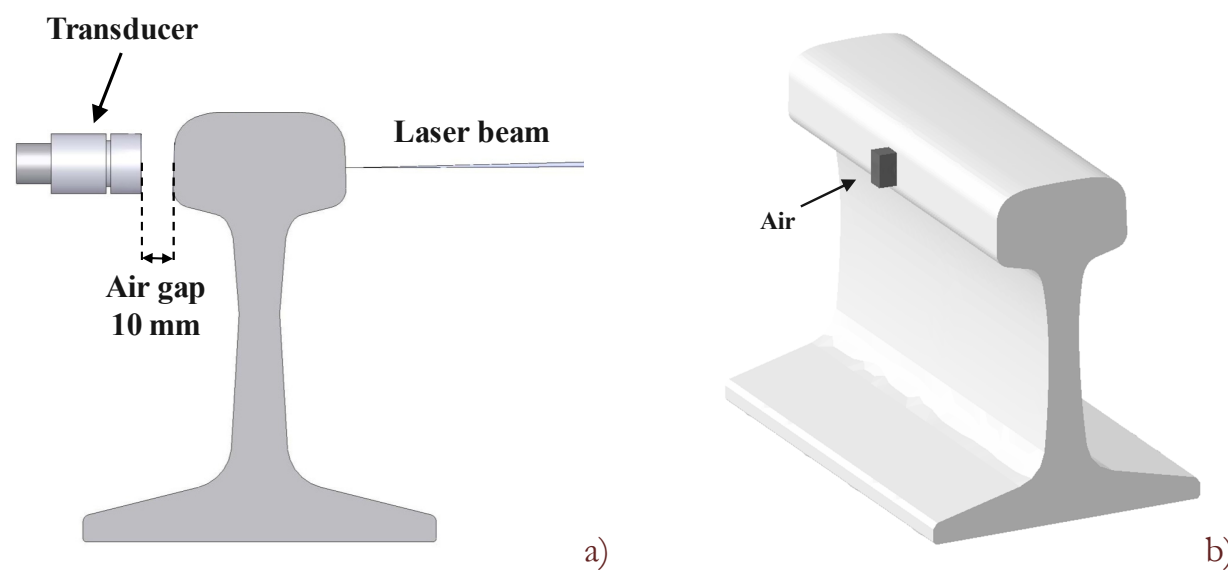

Figura 15: a) Schema del setup sperimentale e b) modello agli elementi finiti della rotaia e della porzione di aria tra la superficie della rotaia e il trasduttore.

Nella Fig. 17a il grafico mostra il segnale acquisito dal sensore piezoelettrico senza contatto. La Fig. 17b presenta la pressione acustica misurata dall'approccio numerico nel punto corrispondente al centro della superficie ricevente del sensore. Il tempo di arrivo della prima onda, indicata con il numero uno nelle Fig. 17a e $17 \mathrm{~b}$, è circa $40 \mu \mathrm{s}$ come trovato precedentemente dalla somma del tempo impiegato dall'onda longitudinale per attraversare il fungo e la porzione di aria. Dopo l'arrivo del primo gruppo di onde, viene registrato, sia dagli esperimenti che dall'analisi numerica, un gruppo di 
onde più forti, indicato con il numero due nelle Fig. 17a e 17b. Queste onde con pressione acustica elevata erano già state viste nell'intervallo da $55 \cdot 10^{-6}$ a $70 \cdot 10^{-6}$ secondi nelle Fig. 16 da d) ad f).

Risultati sperimentali e numerici si corrispondono piuttosto bene anche con l'impiego del trasduttore senza contatto, mostrando l'efficienza del metodo nel simulare la generazione delle onde tramite laser, nel range dei $\mathrm{MHz}$, e la propagazione in acciaio e in aria.

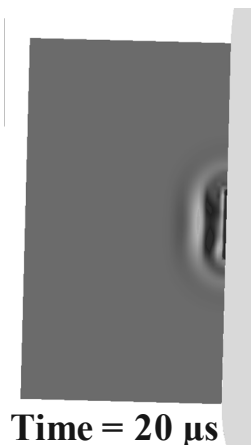

a)

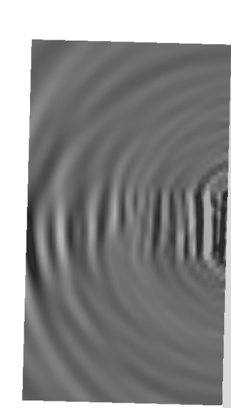

Time $=70 \mu \mathrm{s}$

f)

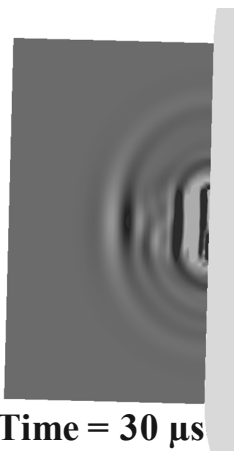

b)

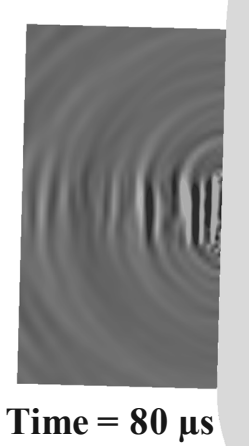

g)

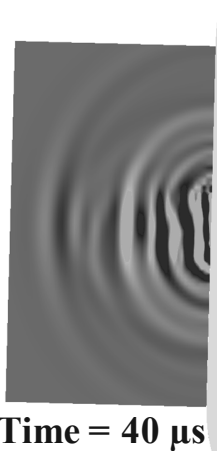

c)

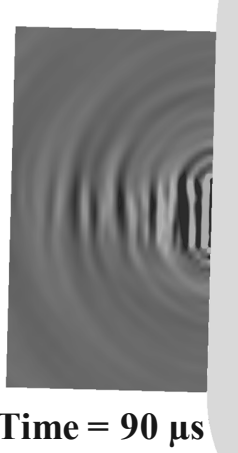

h)

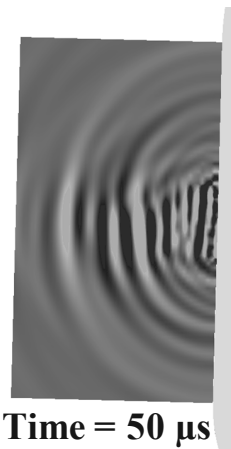

d)

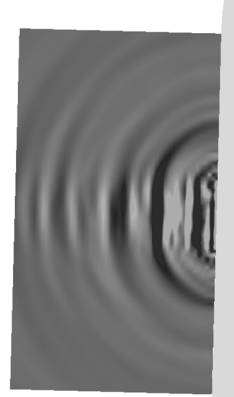

Time $=100 \mu \mathrm{s}$

i)

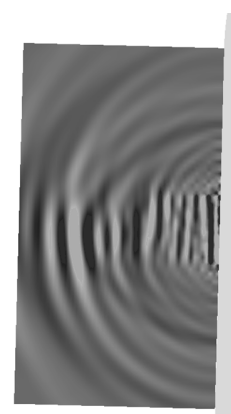

Time $=60 \mu \mathrm{s}$

e)

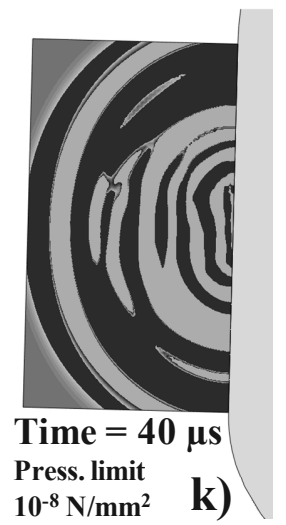

Figura 16: Mappe di pressione acustica nella sezione centrale della porzione di aria tra la superficie della rotaia e il trasduttore in nove istanti differenti.
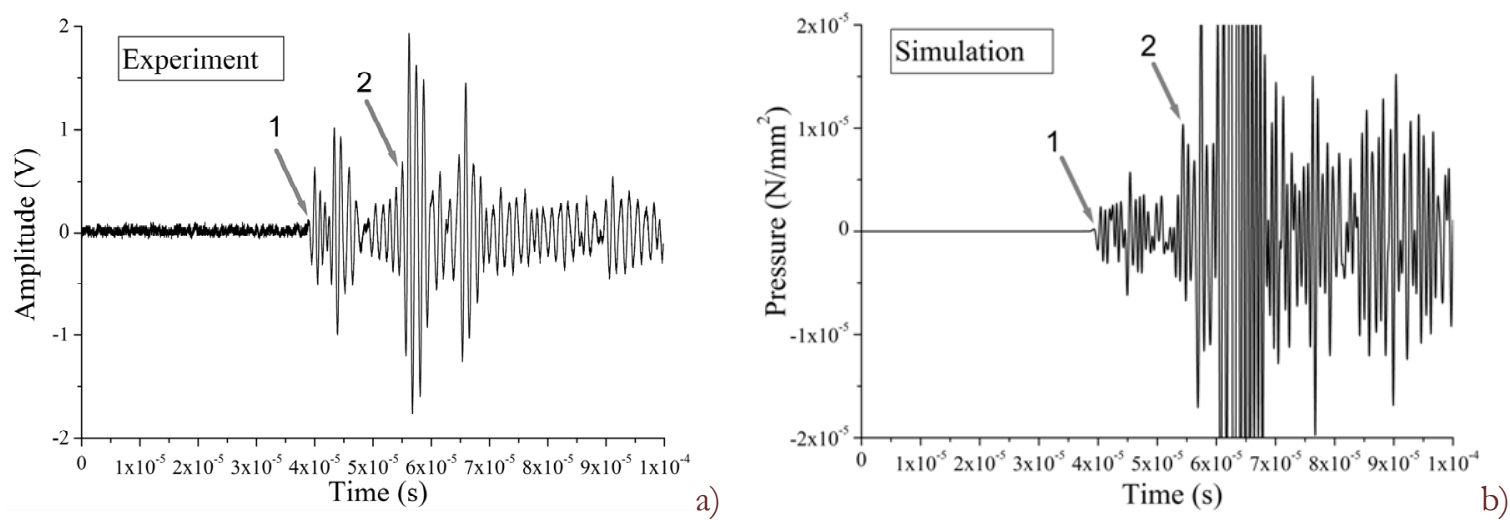

Figura 17: a) Tensione elettrica, proporzionale alla pressione dell'aria, in funzione del tempo per il trasduttore posizionato sul lato del fungo; b) pressione dell'aria misurata con l'approccio numerico nello stesso punto.

Nel paragrafo delle procedure di modellazione è stata discussa la necessità di imporre la condizione di contorni non riflettenti sulle superfici della porzione di aria che non sono in contatto con il fungo della rotaia. Nelle Fig. 18 da a) ad e) sono riportate le mappe di pressione acustica in assenza di condizioni al contorno di non riflessione per l'intervallo da 
$50 \cdot 10^{-6}$ a $90 \cdot 10^{-6}$ secondi. Come si può notare vengono generate un gran numero di onde riflesse; le riflessioni ai contorni che non sono presenti nella realtà possono influenzare l'accuratezza della simulazione.

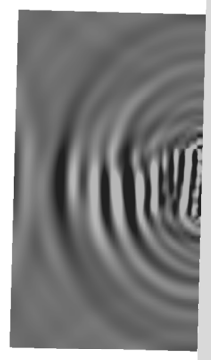

Time $=\mathbf{5 0} \mu \mathrm{s}$

a)

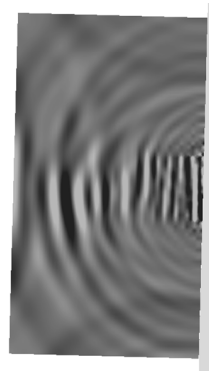

Time $=60 \mu \mathrm{s}$

b)

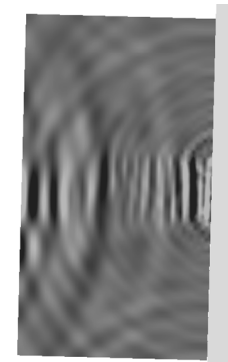

Time $=70 \mu s$

c)

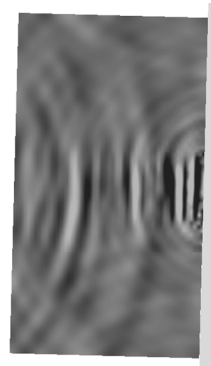

Time $=80 \mu s$

d)

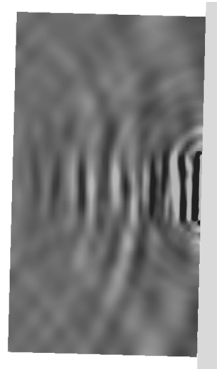

Time $=90 \mu s$

e)

Figura 18: Mappe di pressione nella sezione centrale della porzione di aria tra la superficie della rotaia e il trasduttore per il modello in cui è imposta la condizione di contorno riflettente nell'intervallo di tempo da $50 \cdot 10^{-6}$ a $90 \cdot 10^{-6}$ secondi.

\section{CONCLUSIONI}

$\mathrm{N}$

onostante il potenziale impatto dell'uso degli ultrasuoni generati tramite laser in molte aree dell'industria, mancano ancora strumenti affidabili per studiare il fenomeno. La propagazione di onde ultrasonore generate tramite laser in strutture di forma non regolare è un fenomeno difficile da analizzare. Le tecniche numeriche reperibili in letteratura mostrano limiti di applicabilità per frequenze nel campo dei MHz e lunghezze d'onda molto corte. In questo lavoro si è stabilito che l'integrazione esplicita delle equazioni del moto nelle analisi dinamiche, invece dell'integrazione implicita comunemente utilizzata, in combinazione con l'utilizzo dello schema a masse concentrate nei nodi, che rende la matrice di massa diagonale, consente di risolvere accuratamente ed efficientemente problemi di propagazione di onde ultrasonore con frequenze nel campo dei $\mathrm{MHz}$ che viaggiano in corpi relativamente grandi e in aria. La simulazione della propagazione delle onde in aria consente di considerare la presenza di trasduttori non a contatto in ricezione così da poter studiare l'intero setup sperimentale. La quantità di aria da considerare nell'analisi numerica deve essere limitata a causa dell'elevatissimo costo computazionale; un modo di risolvere il problema è di implementare condizioni al contorno di non riflessione. La metodologia numerica proposta consente di studiare la propagazione di ultrasuoni e di ottimizzare la configurazione dei controlli non distruttivi.

L'approccio numerico è stato convalidato tramite confronto con risultati analitici relativi ad onde guidate che si propagano in un anello circolare. Successivamente si è focalizzata l'attenzione sull'efficienza della tecnica numerica presentata nel simulare i controlli non distruttivi, per rilevare la presenza di difetti nelle rotaie, effettuati utilizzando onde ultrasonore generate tramite laser.

Si è trovato un buon accordo tra i risultati numerici e quelli sperimentali.

\section{BIBLIOGRAFIA}

[1] M. C. Bhardwaj: in: Encyclopedia of Smart Materials, John Wiley \& Sons, New York, (2001).

[2] E. Blomme, D. Bulcaen, F. Declercq, Ultrasonics, 40 (2002) 153.

[3] C.B. Scruby, L.E. Drain, Laser Ultrasonics: Techniques and Applications, Adam Hilger (1990).

[4] S. Kenderian, B.B. Djordjevic, D.Cerniglia, G. Garcia, Insight, 48 (2006) 336.

[5] S. Kenderian, D. Cerniglia, B. B. Djordjevic, R. E. Green Jr., Research in Nondestructive Evaluation, 16 (2005) 195.

[6] R. White, J. Appl. Phys., 34 (1963) 3559.

[7] C.B. Scruby, R.J. Dewhurst, D.A. Hutchins, S.B. Palmer, J. Appl. Phys., 51 (1980) 6210.

[8] L.R.F. Rose, J. Acoust. Soc. Am., 75 (1984) 723.

[9] F.A. McDonald, Appl. Phys. Lett., 56 (1990) 230.

[10] L. Gavric, Journal of Sound and Vibration, 185 (1995) 531.

[11] F. Moser, L.J. Jacobs, J. Qu, NDT\&E International, 32 (1999) 225.

[12] R Sanderson, S Smith, Insight, 44 (2002) 359. 
[13] W. Hassan, W. Veronesi, Ultrasonics, 41 (2003) 41.

[14] A. Zerwer, M. A. Polak, J. C. Santamarina, Journal of Nondestructive Evaluation, 22 (2003) 2.

[15] B. Xu, Z. Shen, X. Ni, J. Lu, Journal of Applied Physics, 95 (2004) 2116.

[16] S. Zhou, P. Reynolds, R. Krause, T. Buma, M. Donnell, J.A. Hossack, Ultrasonics, Ferroelectrics and Frequency Control IEEE Transactions, 51 (2004) 1178.

[17] H. Jeong, M.C. Park, Research in Nondestructive Evaluation, 16 (2005) 1.

[18] J. Wang, Z. Shen, B. Xu, X. Ni, J. Guan, J. Lu, Appl. Phys. A, 84 (2006) 301.

[19] B. Xu, Z. Shen, J. Wang, X. Ni, J. Guan, J. Lu, J. Appl. Phys., 99 (2006) 33508.

[20] E. Glushkov, N. Glushkova, A. Ekhlakov, E. Shapar, Wave Motion, 43 (2006) 458.

[21] I. Bartoli, A. Marzania, F. Lanza di Scalea, E. Viola, Journal of Sound and Vibration, 295 (2006) 685.

[22] N. Terrien, D. Royer, F. Lepoutre, A. Deom, Ultrasonics, 46 (2007) 251.

[23] R. Cook, D. Malkus, M. Plesha, Concepts and Applications of Finite Element Analysis, John Wiley \& Sons, New York, (1989).

[24] G. Liu, J. Qu, J. Acoust. Soc. Am., 104 (1998) 1210.

[25] American Railway Engineering and Maintenance-of-Way Association, AREMA Manual for Railway Engineering, AREMA, Lanham MD USA (2000).

[26] A. Pantano, D. Cerniglia, Applied Physics A: Materials Science \& Processing, 91 (2008) 521. 